\title{
Influence Across Agents and Issues in Combinatorial and Collective Decision-Making
}

\author{
Hang Luo $\left.{ }^{(}\right)(10$ \\ Peking University, Beijing 100871, China \\ hang.luo@pku.edu.cn
}

\begin{abstract}
We consider settings of combinatorial and collective decisionmaking where a set of agents make choices on a set of issues in sequence based on their preferences over a set of alternatives for each issue. While agents have their initial preferences on issues, they may influence others and be influenced by others, consequently changing their preferences or choices on these issues in the process of decision-making. Though the influence among multiple agents making decisions on one issue and the dependency (influence) among multiple issues decided by one agent have been fully discussed in previous work, the influence from multiple sources across both agents and issues in the context of combinatorial and collective decision-making has been ignored. In this paper, we proposed a preliminary framework to address the influence transcending multiple agents and multiple issues with two rules: weighted influence and one dominant influence.
\end{abstract}

Keywords: Combinatorial and collective decision-making - Influence across agents and issues $\cdot$ Weighted influence $\cdot$ One dominant influence

\section{Introduction}

We consider settings of combinatorial and collective (namely, multi-issue and multi-agent) decision-making, where a set of agents (a general term that can represent a person or an artificial intelligence in nature and that can represent a decision-maker, a voter, or a game player in function, etc.) make choices pertaining to a set of issues in sequence based on their preferences regarding a set of alternatives for each issue. While agents have their initial preferences on a series of issues, they may interact with each other, be fully motivated to influence others, and, accordingly, be influenced by others, consequently changing their preferences and ultimately their choices on these issues in the process of decisionmaking [32]. The influence on preferences or choices is achieved via the exchange and diffusion of information among agents and across issues. The information that agents exchange can be from other agents' observable (decision-making) behaviors [32] or from others agents' declared or shared preferences (underlying their choices) [25].

(C) Springer Nature Switzerland AG 2020

D. C. Morais et al. (Eds.): GDN 2020, LNBIP 388, pp. 75-90, 2020.

https://doi.org/10.1007/978-3-030-48641-9_6 
Actually, the interaction and influence among agents while making decisions is quite common in reality, and has been studied by scholars from multiple disciplines [25,26], including computer science and artificial intelligence (particularly multi-agent system) $[5,17,25,26,32,33,35,37]$, economics and management science (particularly decision theory and social network) [7-16, 19, 20,23, 24,34], and even politics [28]. For example, in international politics, the decision-making process of the United Nations (UN) Security Council entails various influences among member states, including both positive influences among allies and negative influences among opponents. Each member state in the UN Security Council has full motivation to convince and influence other member states' votes in order to gain desired voting results and maximize its own state interests. Member states in the same alliance usually support each other, therefore positively influencing each other. For instance, the United Kingdom usually casts the same votes as the United States. However, member states from confronting camps usually oppose each other, thereby negatively influencing each other. For instance, the former Soviet Union ${ }^{1}$ and present-day Russia usually veto the draft resolutions proposed by the United States.

Moreover, the dependency (influence) among issues for decision-making is also very common in reality, and has first drawn attention from computer scientist $[2,3,18,36,38,39]$. When an agent is making decisions on a series of issues, it is natural for him/her to refer to his/her own choices regarding the same or similar issues in the past. Namely, an agent's preferences/choices on later issues are usually dependent on (or affected by) his/her own choices on prior issues. There are both positive and negative dependencies among issues. An agent will positively reference (usually, make the same decision as) his/her satisfactory choices in the past but will negatively reference (usually, make the opposite decision to) his/her regrettable choices from the past. For example, in international politics, the United States always used its veto power on the draft resolutions to punish Israel in the UN Security Council, not only for its own state interests but also for its reputation in the international community and particularly in the mind of its allies (a great power should be constant and trustworthy regarding its attitudes toward critical issues and provide a stable expectation for its allies).

\section{Related Works}

Most previous works either studied the influence among multiple agents while making decisions on a single issue (usually, in the framework of social networks) $[5,8-17,19,20,23-26,34,35,37]$ or studied the dependency among multiple issues decided by a single agent (typically, using the model of CP-nets, namely, conditional preference networks) $[2,3,18,36,38,39]$. A few studies have combined the influence among multiple agents and the dependency among multiple issues $[32,33]$ in the same model, but they still just discussed them separately and did not study the influence across both agents and issues. Specifically, this means

\footnotetext{
${ }^{1}$ During the first 10 years of the United Nations, the former Soviet Union representative, Andrei Gromyko, even had the nickname "Mr. No".
} 
that there are horizontal influences among agents making decisions on a single issue and vertical dependencies among issues decided by a single agent, but there is no diagonal influence across multiple agents and multiple issues (as shown in Fig. 1) discussed.

It should be noted that traditionally, the dependency among issues would not be deemed the influence, but in fact, the dependency among issues could be understood as a "special" kind of influence. The dependency among issues means that the preferences/choices of an agent on later issues will be affected by his/her own choices on former issues, in a sense, such "affected" just means "influenced". Based on and improving upon the models of $[32,33]$, we build a framework to model the influence across multiple agents and multiple issues, where agents express their preferences as CP-nets, and influences (dependencies) among agents (issues) are expressed as directed links in networks. Before officially representing this framework, we introduce related works on the study of influence in combinatorial and collective decision-making using Example 1, as follows:

Example 1. (UN Security Council Decision-making) This is a typical example of combinatorial and collective decision-making with influences among agents (member states) and dependencies among issues (draft resolutions).

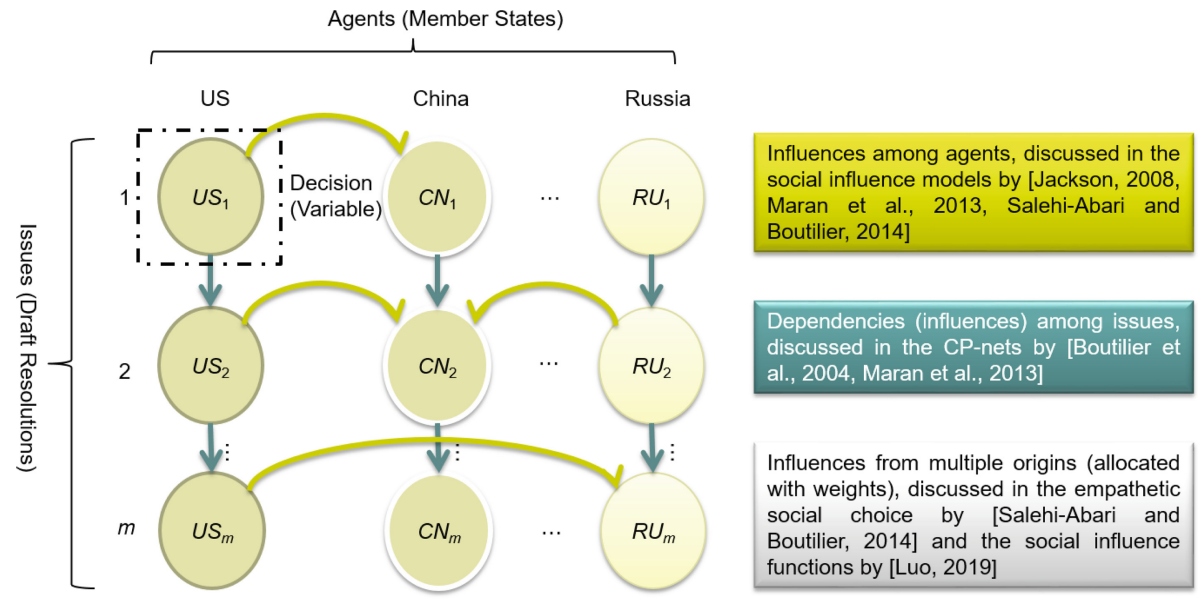

Fig. 1. The UN security council as a typical combinatorial and collective decisionmaking with influences among agents and dependencies among issues (Notes: Green links represent the dependencies (influences) among issues (draft resolutions) decided by a single agent (member state), and yellow links represent the influences among agents (member states) making decisions on a single issue (draft resolution).) (Color figure online)

First, the UN Security Council decision-making is a typical multi-agent (collective) decision. There are 15 member states (including 5 permanent members and 10 nonpermanent members) collectively making decisions for each draft 
resolution. Each member state tries to persuade (represented as positively influencing) its allies ${ }^{2}$ and opposes (represented as being negatively influenced by) its opponents, in order to pursue its own state interests and achieve desirable voting results on critical international issues. It is easy to see that the UN Security Council is full of varied games, interactions and persuasions among member states, which means that there are influences (expressed as the horizontal directed links shown in Fig. 1) among agents (member states). The models describing the influence among agents are mainly the social influence models $[5,8-17,19,20,25,26,35,37]$.

Second, the UN Security Council decision-making is also a typical multi-issue (combinatorial) decision. The Council has made decisions on thousands of draft resolutions since the establishment of the UN. Moreover, there are many draft resolutions frequently addressing the same subject (such as the Israel-Palestine issue, Iraq issue and Syria issue). Usually, the votes of a given member state on later draft resolutions would be affected by (refer to) its own votes on former draft resolutions with the same or similar subjects, which means that there are dependencies (represented as the vertical directed links shown in Fig. 1) among issues (draft resolutions). The typical framework describing the dependency among issues is the CP-nets $[2,3,18,36,38,39]$.

Third, it should be noted that the influences among member states do not simply flow from one to another but usually simultaneously from multiple ones, which will make the process of influence and the determination of the results of influence complicated. For instance, a vote of China may be influenced by Russia, the United States and some other states at the same time. As such, how do we address the multiple sources of influence and determine the resulting preference or choice, especially in light of contradictive influencing directions (such as a positive influence from Russia and a negative influence from the United States) and diversified influencing weights (such as a stronger influence from a great power and a weaker influence from a small country)? [25] Models addressing multiple sources of influence include the empathetic social choice [37], social influence functions [25] and so on.

In fact, influence in present-day society has become much more intensive, particularly with the large-scale online communication via the Internet beyond the limitation of space, time and environment [22-24,27,31,34]. More specifically, with the advancement of wireless network technology and mobile communication devices, particularly with the help of online social platforms, such as Facebook ${ }^{\circledR}$, Twitter ${ }^{\circledR}$, and WeChat ${ }^{\circledR}$, interaction and communication among people (particularly those in remote locations) have become much more convenient and frequent than before [22-24,27,31,34]. If you like, you can instantly communicate with others overseas. Keeping in touch with friends who are far away is no longer a problem in the sense of space, time and environment. Thus, current studies in decision-making should typically involve a very large number of agents interacting with each other and making decisions on a series of issues

\footnotetext{
${ }^{2}$ To let them support what it supports, or oppose what it opposes.
} 
as opposed to restricted cases consisting of a few agents making decisions on a single issue, or a few issues decided by a single agent. In this context, not only should the preference/choice of each single agent for each single issue be discussed, but also the interaction and influence among different agents on different issues should be fully investigated, which makes the study of psychology and behavior of a decision-maker, the mechanisms and dimensions of influence, and the aggregation for collective preference/choice in group decision-making much more complicated. ${ }^{3}$

Moreover, it is not that simple as we might expect even for the influence just among multiple agents (while making decisions on a single issue), as the influence of reality faced by an agent usually comes not from a single agent at a time but from more than one agent at the same time $[25,26]$. There are a series of approaches to address multiple sources of influence in group decision-making:

\subsection{How to Address Multiple Sources of Influence Among Agents in a Cardinal Approach}

Multiple sources of influence in group decision-making have been preliminarily discussed by Salehi-Abari and Boutilier [37] as an empathetic social choice model in the environment of social networks, setting a weight of influence for each influencing agent, in which an agent's utility value is collectively affected by both other agents' utilities and his/her own initial utility. As both the subject of influence and the object of influence are utility values of agents, it is a cardinal (utility value-based) approach. Moreover, it assumes all influence as positive, which may be oversimplifying compared with reality. It is certain that there are positive influences from friends, families, or relatives and so on; however, it is also impossible to avoid all negative influences from enemies, opponents, or any person with a negative appreciation in real-world settings [25].

\subsection{How to Address Multiple Sources of Influence Among Agents in an Ordinal Approach}

Luo [25] further discussed how to address multiple sources of influence via an ordinal (preference ordering-based) approach and extended the KSB metric $[1,21,38,39]$ to a weighted and signed matrix influence function in the context of group decision-making with mutual influence, in which the weight of influence could be stronger or weaker in strength, and positive or negative in polarity.

\subsection{From Social Choice Functions to Social Influence Functions}

Luo [25] also extended several classical social choice functions, including nonranked choice methods such as the plurality and the majority and ranked

\footnotetext{
${ }^{3}$ Compared with a set of agents independently making choices, combinatorial and collective decision-making with mutual influence entails far more than the simple (linear) aggregation of independent preferences/choices of agents.
} 
choice methods such as the Borda count and the Condorcet method, to signed and weighted social influence functions, respectively as: plurality influence rule, majority influence rule, Borda influence rule and Condorcet influence rule.

However, all these works mainly studied how to address multiple sources of influence among agents' preferences or choices just on a single issue. Overall, the influence from more than one agent making a decision on one issue (namely, the multiple influences in the horizontal dimension) has been fully discussed; besides, the dependency on (influence from) more than one issue for one agent's decisionmaking (namely, the multiple influences in the vertical dimension) has been preliminarily described in the model of [18]; however, the influence across both more than one agent and more than one issue (namely, the multiple influences in the diagonal dimension) has been ignored.

There is a general meaning for the study of influence across agents and issues in combinatorial and collective decision-making, not only with regard to theory (of computer science, artificial intelligence, social choice and group decision and so on ${ }^{4}$ ) but also as it pertains to application (of joint-stock company voting, political elections, international organization decision-making ${ }^{5}$ and so on).

\section{Multiple Sources of Influence Across Agents and Issues}

Previous studies have mainly discussed the influence from multiple agents while making decisions on a single issue or the dependency on (influence by) multiple issues for a single agent's decision-making. However, an agent's preference/choice on an issue could be simultaneously influenced by multiple agents' preferences/choices on multiple issues. Thus, the origin of influence is a more general entity, involving both agents and issues. We propose a framework of combinatorial and collective decision-making with influence across multiple agents and multiple issues using Example 2, as follows:

Example 2. (A General Example of Influences across Agents and Issues) Assume a multi-agent and multi-issue decision-making case with a set of agents $\{1,2,3\}$ making choices on a set of issues $\{X, Y, Z\}$, each with three alternatives, as shown in Fig. 2. While agent 3 is making a decision on issue $Z$, it is possible that his/her preference/choice will be influenced by agent 2's preference/choice on the same issue $Z$ (which is an influence between agents making decisions on a single issue) and his/her own preference/choice on former issue $Y$ (which

\footnotetext{
${ }^{4}$ It is specially meaningful in the field of computational social choice $[4,6]$, which is an interdisciplinary research field of computer science (particularly artificial intelligence) and social choice theory. Specifically, it is the study of social choice from the perspective of computer science.

${ }^{5}$ Nearly all international organizations adopt typical group decision-making systems, regardless of whether it is for international political organizations such as the UN General Assembly, UN Security Council [28] and Council of the European Union or international economic organizations such as the World Bank, IMF, Asian Infrastructure Investment Banks [29], and New Development Bank [30].
} 
is a dependency between issues decided by a single agent), while at the same time, influenced by agent 2's preference/choice on former issue $Y$ and agent 1's preference/choice on former issue $X$ (which are influences across both agents and issues).

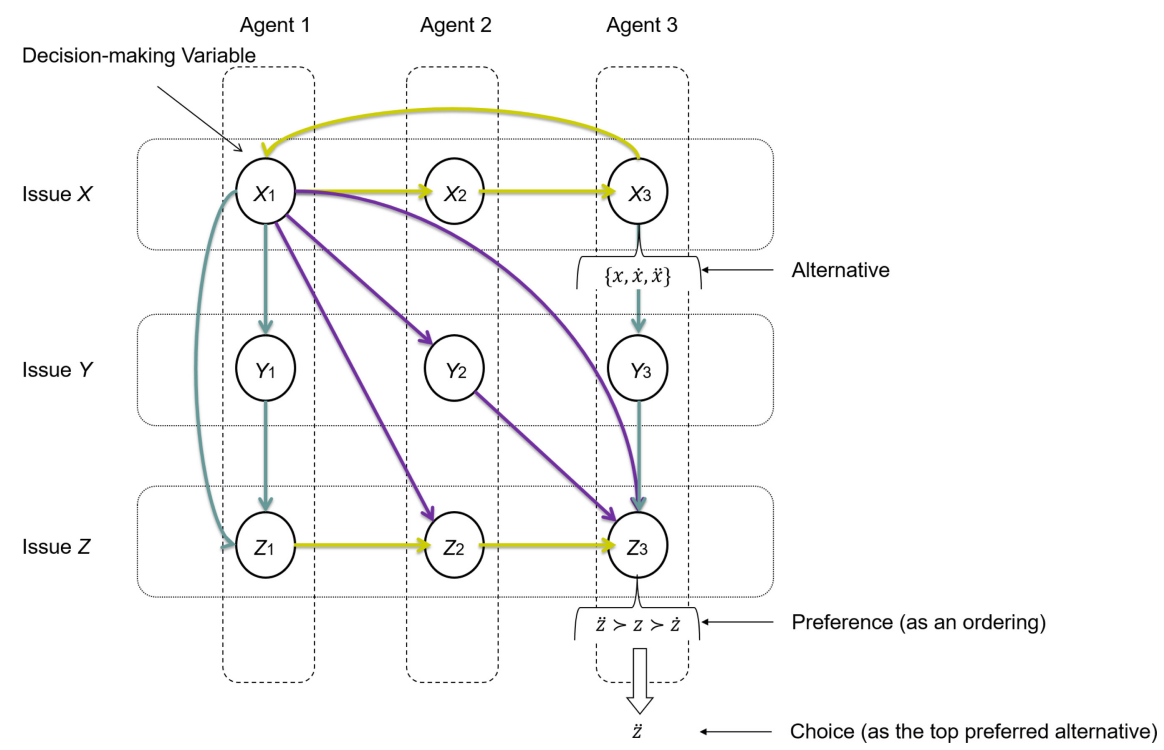

Fig. 2. Influences transcending multiple agents and multiple issues (Notes: Green links represent the dependencies (influences) among multiple issues decided by a single agent; yellow links represent the influences among multiple agents making decisions on a single issue; purple links represent the influences across both agents and issues.) (Color figure online)

When an agent's preference/choice on an issue is simultaneously influenced by more than one agent's preference/choice on more than one issue, especially when each origin of influence has contradictive influencing directions (positive or negative) and varied influencing strengths (weaker or stronger), how to determine the result of the collective influence is an important but not discussed issue. It is relatively easy to set the rule of influence from multiple agents to another agent while making a decision on a single issue or the rule of dependency on multiple (former) issues of another (later) issue decided by a single agent, but it is much more complicated to design a rule of influence from more than one origin across both agents and issues.

In fact, the influence from multiple origins across agents and issues is very common in real-world situations. It is oversimplifying to assume that each agent's preference/choice on each issue is influenced by other agents' preferences/choices only on the same issue, or influenced only by his/her own preferences/choices 
on other former issues, or only influenced by one other agent's preference/choice on one other issue at a time (or in a round). The reality is that a person's preference/choice on an issue can be influenced by different people (who can be friends, families and so on)'s preferences/choices on different issues to different extents at the same time.

Example 3. (A Specific Example of Stronger and Weaker Influences across Agents and Issues: a Family Buying a Car) Assume a family is choosing a car, and it is a "democratic" instead of "dictatorial" family that all families members have voices. There may be a case that the preference of the husband on the mode of the car will be heavily influenced by his wife's preference or choice regarding the manufacturer of the car: if his wife wants a BMW ${ }^{\circledR}$, he may be much more inclined to buy a commercial car than a SUV, but if his wife wants a Jeep ${ }^{\circledR}$, he may be much more inclined to buy a SUV than a commercial car; meanwhile, his preference will be slightly influenced by his kids' preferences or choices regarding the color of the car: if his kids want a black car, he may be a little more inclined to buy a commercial car than a sports car, but if his kids want a red car, he may be a little more inclined to buy a sports car than a commercial car.

Moreover, a person's preference/choice on an issue can also be simultaneously positively influenced by some people (usually as friends)'s and negatively influenced by some other people (usually as enemies)'s preferences/choices on different issues.

Example 4. (A Specific Example of Positive and Negative Influences across Agents and Issues: the UN Security Council Voting) During the process of the UN Security Council voting, there are both positive influences and negative influences among member states on a series of issues (draft resolutions) due to the existence of conflicting state interests and confronting alliances (camps) ${ }^{6}$. The vote of a member state on a draft resolution may be positively influenced by the preferences of its allies on the same draft resolution or the votes of its allies on former relevant draft resolutions (with the same or similar subjects) and negatively influenced by the preferences of its opponents on the same draft resolution or the votes of its opponents on former relevant draft resolutions.

In a multi-agent and multi-issue decision-making context, each influencing and influenced entity, which can be defined as decision-making variables (typically as preference and choice), needs two coordinates (one is the issue-coordinate and the other is the agent-coordinate) to be located, namely, to know which agent is making a decision and on which issue (as shown in Fig. 2). We define the decision-making variables and some background variables in a combinatorial and collective decision-making context as follows:

\footnotetext{
${ }^{6}$ Such as the confrontation between the NATO led by the United States and the Warsaw Pact led by the former Soviet Union in the history and the antagonism between the NATO and Russia and its allies nowadays.
} 
Definition 1. (Combinatorial and Collective Decision-making Society with Influence Across Agents and Issues) Assume a society $\mathbb{S}=\{\mathbb{N}, \mathbb{I}, \mathbb{P}, \mathbb{C}, \mathbb{W}\}$ : $\mathbb{N}=\{1,2, \ldots, n\}$ is the set of all agents; $\mathbb{I}=\left\{I_{(1)}, I_{(2)}, \ldots, I_{(m)}\right\}$ is the set of all issues (or features of a multiple-feature issue); then, there are $n \times m$ decisionmaking variables ( $n$ agents times with $m$ issues) in total; $\mathbb{P}=\left\{P_{(1)}(1), P_{(1)}(2)\right.$, $\left.\ldots, P_{(1)}(m), P_{(2)}(1), P_{(2)}(2), \ldots, P_{(2)}(m), \ldots, P_{(n)}(1), P_{(n)}(2), \ldots, P_{(n)}(m)\right\}$ is the set of all agents' preferences (such as preference orderings, utilities, beliefs, opinions, decision-making probabilities, etc.) on all issues; $\mathbb{C}=\left\{C_{(1)}(1), C_{(1)}(2)\right.$, $\left.\ldots, C_{(1)}(m), C_{(2)}(1), C_{(2)}(2), \ldots, C_{(2)}(m), \ldots, C_{(n)}(1), C_{(n)}(2), \ldots, C_{(n)}(m)\right\}$ is the set of all agents' choices on all issues, namely, counting from agent 1 's preference/choice on issue 1 to agent n's preference/choice on issue $m$, in which $P_{(i)}(q)$ represents the preference of agent $i$ on issue $q, C_{(i)}(q)$ represents the choice of agent $i$ on issue $q(i \in \mathbb{N}, q \in \mathbb{I}) ; \mathbb{W}$ is the matrix whose entries are the weights of influence between each of two decision-making variables, $\mathbb{W}=\left[w_{(i, j)}(q, h)\right]$ $(i, j \in \mathbb{N}, q, h \in \mathbb{I})$, in which $w_{(i, j)}(q, h)$ reflects the weight of influence from agent $i$ 's preference/choice on issue $q$ to agent $j$ 's preference/choice on issue $h$, the weight value indicates both the strength and polarity of the influence, $w_{(i, j)}(q, h)>0$ means a positive influence, $w_{(i, j)}(q, h)<0$ means a negative influence, and $w_{(i, j)}(q, h)=0$ means there is no influence from agent $i$ 's preference/choice on issue $q$ to agent $j$ 's preference/choice on issue $h$; besides, the higher $\left|w_{(i, j)}(q, h)\right|$ is, the stronger is the influence from agent $i$ 's preference/choice on issue $q$ to agent $j$ 's preference/choice on issue $h$.

In this paper, two preliminary rules: weighted influence and one dominant influence, addressing the influence from more than one origin across agents and issues, are constructed from different perspectives. We first provide a simple comparison of the two rules when it is for the influence among multiple agents while making decisions just on a single issue, as shown in Example 5, then extend them to a multi-agent and multi-issue decision-making context.

Example 5. (Weighted Influence vs. One Dominant Influence among Agents Making Decisions on a Single Issue) Assume a multi-agent decision-making case with 8 agents making choices on one issue with the set of alternatives as: $\{a, b, c\}$, as shown in Fig. 3. While the agent in the middle is making a decision on this issue, he/she is simultaneously influenced by all other agents possessing various preference orderings with diversified weights of influence, in which some influences are positive, some other influences are negative, and some influences are stronger than others and even with 3 times of strength. However, all influences are from multiple agents but toward a single issue's decision. The comparison of how the two rules address the influence across both agents and issues will be discussed in details in following. 

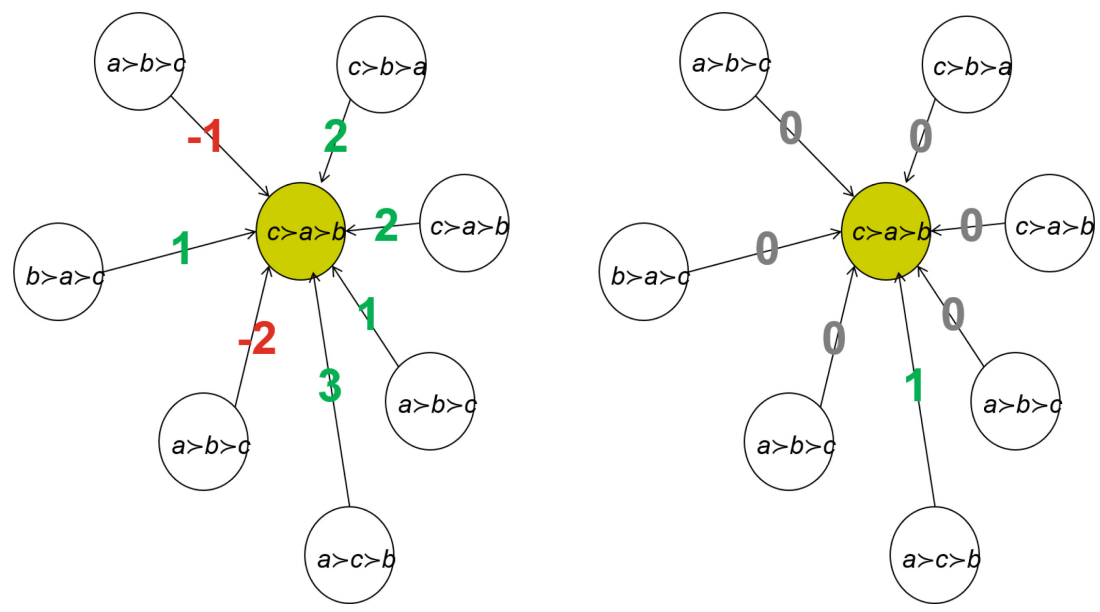

Fig. 3. Weighted influence vs. One dominant influence among multiple agents making decisions on a single issue

\section{Weighted Influence Across Agents and Issues}

One rule for multiple influences across agents and issues is to assume that the preference/choice (of an agent on an issue) can be collectively influenced by all of the influencing preferences/choices (of more than one agent and on more than one issue), just to different extents and directions according to their respective weights of influence. Actually, the use of the weight of influence is a traditional approach to address the influence among agents in group decision-making and has been much discussed in previous works such as $[20,25,26,37]$. In the empathetic social choice model [37] built via a cardinal approach, the utility value of the influenced agent is the weighted sum of utilities of all influencing agents (including himself/herself and his/her "neighbors"). Luo [25] extended classical social choice functions, such as the Borda count and the Condorcet method, to signed and weighted social influence functions, which means that both positive and negative influences and both stronger and weaker influences (from all influencing agents) are collectively handled to obtain the resulting choice (of the influenced agent). Moreover, Luo [25] extended the KSB metric [1,21,38,39] to a signed and weighted matrix influence function to address the multiple sources of influence among agents via an ordering-based approach; he first defined the rule of how to transform each preference ordering (including both the preference orderings held by the influencing agents and the preference orderings existing in theory $)^{7}$ into a matrix and then set a distance metric to compute the distance between any two ordering matrices; the feasible preference ordering that has the smallest weighted sum of distances from all influencing agents' preference

\footnotetext{
${ }^{7}$ If there are $m$ alternatives (candidates), then all the possible preference orderings over them would be $m$ ! kinds.
} 
orderings will then be the resulting preference (of the influenced agent) [25,26]. As the weight of agents' influences can be either positive or negative (namely, as friends or enemies) in real-world settings, it will partially play a role in finding the "closest" possible preference from the positively influencing agents' preferences and partially play a role in finding the "farthest" possible preference from the negatively influencing agents' preferences for the resulting preference [25].

The empathetic social choice [37], social influence functions [25] and matrix influence function [25] and so on all discussed the influence from more than one agent making a decision on a single issue but not from more than one origin across both agents and issues. However, these models all have potentials to be extended to address the influence transcending multiple agents and multiple issues in the context of combinatorial and collective decision-making. To achieve this, a precondition is to build a weight matrix whose entries are the weights of influence from each decision-making variable $C_{i, q}$ to each of the other decisionmaking variables $C_{j, h}(i, j \in \mathbb{N}, q, h \in \mathbb{I})$.

Example 6. (A Display of the Weighted Influence across Agents and Issues) Assume a multi-agent and multi-issue decision-making case with a set of agents $\{1,2,3\}$ making choices on a set of issues $\{X, Y, Z\}$, each with three alternatives, as shown in Fig. 4. From the perspective of agent 3 making a decision on issue $Z$, he/she is simultaneously influenced by three agents including himself/herself making decisions on three issues including issue $Z$. Agent 1 is a friend (ally) in the mind of agent 3, thus, agent 1's preferences/choices on current issue $Z$ and on former issue $X$ produce positive influences (with weights of influence respectively as: 3 and 1 ) on agent 3's preference/choice on issue $Z$. Agent 2 is an enemy (opponent) in the mind of agent 3, thus, agent 2's preferences/choices on current issue $Z$ and on former issue $Y$ produce negative influences (with weights of influence respectively as: -2 and -1 ) on agent 3 's preference/choice on issue $Z$. Furthermore, agent 3's preference/choice on current issue $Z$ is influenced by (dependent on) his/her own preferences/choices on former issue $X$ and $Y$ (with weights of influence respectively as: 3 and 4 ).

Usually, a choice near current time has higher weight of influence than one other choice far from current time, as people's memories fade with time (regardless of whether it is the satisfaction about a good decision in the past or the regret for a bad decision from the past). What's more, it is quite common that people will be influenced not only by others (such as friends and allies, or enemies and opponents) surrounding them but also by themselves [25]. The latter oneself will inevitably be influenced by the former oneself, and the weight of one's own influence is usually positive [25]. Only in some extreme cases, assume a person encounters serious setbacks and totally loses his/her self-confidence, then his/her own influence could change from strong to weak, and even from positive to negative [25]. Such setup of one's own influence can explain why some people are hard to be influenced by others while some other people are easy to be changed because the former kind of people's self-influences may have higher weights than the latter [25]. 


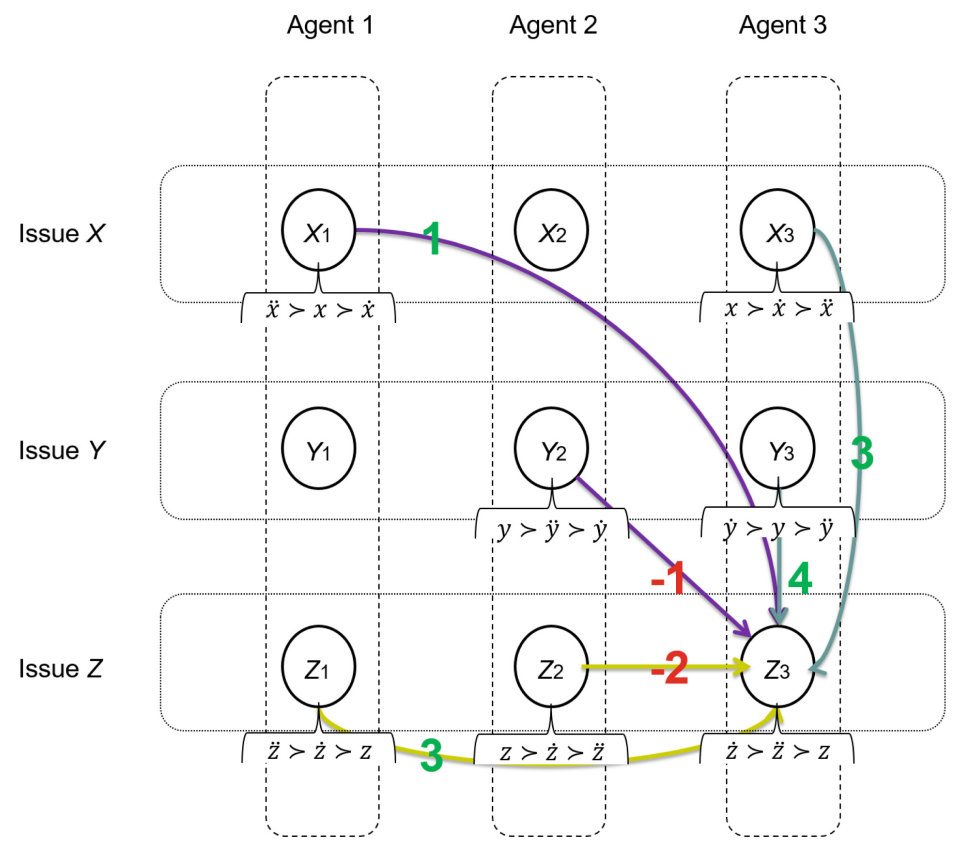

Fig. 4. Weighted influences across agents and issues

\section{One Dominant Influence Across Agents and Issues}

The other rule for multiple influences across agents and issues is to assume that a preference/choice (of an agent on an issue) will just be influenced by the most predominant preference/choice (with the strongest influencing "power") among all of the influencing preferences/choices (of different agents and on different issues). A concept of the priority of influence can be proposed to find the most predominant influencing preference/choice. Assume that there are $n \times m$ decisionmaking variables (namely, $n$ agents making decisions on $m$ issues) and that there is a priority of influence from each decision-making variable $C_{i, q}$ to each of the other decision-making variables $C_{j, h}(i, j \in \mathbb{N}, q, h \in \mathbb{I})$. Thus, each decisionmaking variable will be influenced by the influencing decision-making variable with the highest priority (of influence) on it compared with all of the other influencing decision-making variables. Actually, one simple method to obtain the priority of influence is to connect it to the weight of influence. Specifically, we make the priority of influence between each of two decision-making variables equal to the absolute value of the weight of influence between them. Thus, for each preference/choice being influenced, the influencing preference/choice that has the highest absolute value of the weight of influence on it also has the highest priority of influence on it and can dominate its result of influence. Intuitively, this rule for addressing multiple sources of influence across agents and issues can remarkably reduce the complexity of the computation. 
Moreover, although it is much more simplified compared with weighted influence, in a sense, this rule of one dominant influence may be closer to how people deal with multiple sources of influence across agents and issues in the real-world. For example, while facing a complicated case of multiple influences from many people making decisions on many issues, you will just follow your best friend (or highest leader) on the most important issue for yourself, or just oppose your most hated enemy on the most critical issue for him/her (only if the absolute value of his/her weight of influence on this issue is the highest, regardless of whether it is positive or negative) rather than engage in a complex weighted deliberation and computation.

Example 7. (A Display of the One Dominant Influence across Agents and Issues) Assume a multi-agent and multi-issue decision-making case with a set of agents $\{1,2,3\}$ making choices on a set of issues $\{X, Y, Z\}$, as shown in Fig. 5. Though agent 3 faces complicated multiple influences across both agents and issues while making a decision on issue $Z$, which is the same case as in Example 6, he/she will just follow the influencing decision-making variable with the highest priority of influence (namely, the highest absolute value of the weight of influence here), which is just the preference/choice of himself/herself on former issue $Y$. This way to address multiple sources of influence is just like ignoring all other influences except the most predominant one, in mathematics, as if resetting all other influencing preferences/choices' weights to zero (compared with Fig. 4).

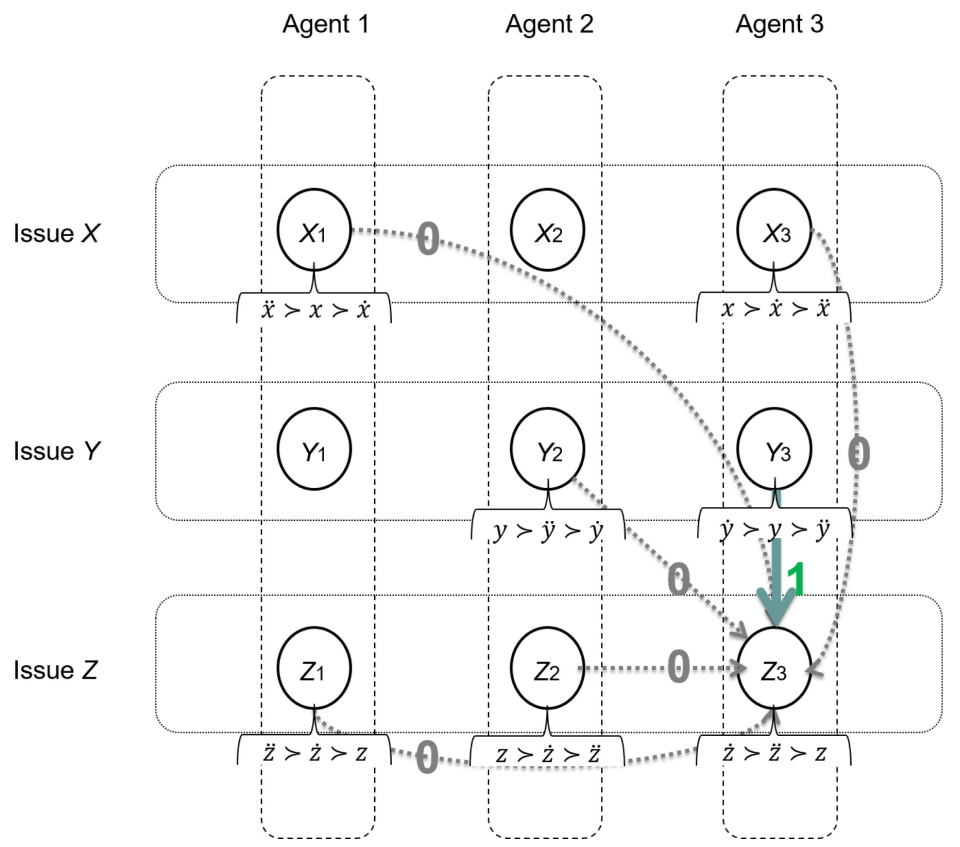

Fig. 5. One dominant influence across agents and issues 


\section{Discussion and Conclusion}

In summary, the study of influence in combinatorial and collective decisionmaking could be advanced according to the following points:

- We need to discuss group decision-making, not just regarding a singe issue or multiple issues independent to each other but pertaining to multiple issues with combinatorial structures of dependencies among them, constituting a combinatorial and collective decision-making context.

- We should not only discuss the simultaneous influence from multiple agents while making decisions on an issue, but also systematically study the simultaneous dependency on (influence by) multiple issues for an agent's decisionmaking, particularly investigate the influence from more than one origin across both agents and issues and address the conflicts among multiple sources of influence with varied strengths and contradictive directions.

- To address the multiple influences across agents and issues, two preliminary approaches of weighted influence and one dominant influence are proposed. In future work, the two approaches could be detailed and improved, and many other promising analytic frameworks and feasible mathematical models could be tried and discussed, particularly considering the case of multiple issues not similar to each other (namely, with different sets of alternatives).

Acknowledgement. This study is supported by a Natural Science Foundation of China Grant (71804006) and a National Natural Science Foundation of China and European Research Council Cooperation and Exchange Grant (7161101045).

\section{References}

1. Bogart, K.P.: Preference structures I: distances between transitive preference relations? J. Math. Sociol. 3(1), 49-67 (1973)

2. Boutilier, C., Brafman, R.I., Domshlak, C., Hoos, H.H., Poole, D.: CP-nets: a tool for representing and reasoning with conditional ceteris paribus preference statements. J. Artif. Intell. Res. 21, 135-191 (2004)

3. Boutilier, C., Brafman, R.I., Domshlak, C., Hoos, H.H., Poole, D.: Preference-based constrained optimization with CP-Nets. Comput. Intell. 20(2), 137-157 (2004)

4. Brandt, F., Conitzer, V., Endriss, U.: Computational social choice. In: Weiss, G. (ed.) Multiagent Systems, pp. 213-283. MIT Press, Cambridge (2013)

5. Capuano, N., Chiclana, F., Fujita, H., Herrera-Viedma, E., Loia, V.: Fuzzy group decision making with incomplete information guided by social influence. IEEE Trans. Fuzzy Syst. 26(3), 1704-1718 (2018)

6. Chevaleyre, Y., Endriss, U., Lang, J., Maudet, N.: A short introduction to computational social choice. In: van Leeuwen, J., Italiano, G.F., van der Hoek, W., Meinel, C., Sack, H., Plášil, F. (eds.) SOFSEM 2007. LNCS, vol. 4362, pp. 51-69. Springer, Heidelberg (2007). https://doi.org/10.1007/978-3-540-69507-3_4

7. Degroot, M.H.: Reaching a consensus. J. Am. Stat. Assoc. 69(345), 118-121 (1974)

8. Demarzo, P.M., Vayanos, D., Zwiebel, J.: Persuasion bias, social influence, and unidimensional opinions. Q. J. Econ. 118(3), 909-968 (2003) 
9. Friedkin, N.E., Johnsen, E.C.: Social influence and opinions. J. Math. Sociol. 15(3-4), 193-206 (1990)

10. Friedkin, N.E., Johnsen, E.C.: Social positions in influence networks. Soc. Netw. 19(3), 209-222 (1997)

11. Golub, B., Jackson, M.O.: Naive learning in social networks and the wisdom of crowds. Am. Econ. J.: Microecon. 2(1), 112-149 (2010)

12. Grabisch, M., Rusinowska, A.: A model of influence in a social network. Theory Decis. 69(1), 69-96 (2010). https://doi.org/10.1007/s11238-008-9109-z

13. Grabisch, M., Rusinowska, A.: A model of influence with an ordered set of possible actions. Theory Decis. 69(4), 635-656 (2010). https://doi.org/10.1007/s11238-0099150-6

14. Grabisch, M., Rusinowska, A.: Measuring influence in command games. Soc. Choice Welfare 33(2), 177-209 (2009). https://doi.org/10.1007/s00355-008-0350-8

15. Grabisch, M., Rusinowska, A.: Influence functions, followers and command games. Games Econ. Behav. 72(1), 123-138 (2011)

16. Grabisch, M., Rusinowska, A.: A model of influence based on aggregation function. Math. Soc. Sci. 66(3), 316-330 (2013)

17. Grandi, U., Lorini, E., Perrussel, L.: Propositional opinion diffusion. In: Proceedings of the 14th International Conference on Autonomous Agents and Multiagent Systems, pp. 989-997 (2015)

18. Grandi, U., Luo, H., Maudet, N., Rossi, F.: Aggregating CP-nets with unfeasible outcomes. In: O'Sullivan, B. (ed.) CP 2014. LNCS, vol. 8656, pp. 366-381. Springer, Cham (2014). https://doi.org/10.1007/978-3-319-10428-7_28

19. Hoede, C., Bakker, R.: A theory of decisional power. J. Math. Sociol. 8, 309-322 (1982)

20. Jackson, M.O.: Social and Economic Networks. Princeton University Press, Princeton (2008)

21. Kemeny, J.G., Snell, J.L.: Mathematical Models in the Social Sciences. The MIT Press, Cambridge (1972)

22. Luo, H.: Multi-agent simulation research on urban agglomeration integration and local government interaction. J. Dalian Univ. Technol. 34(2), 46-52 (2013). in Chinese

23. Luo, H.: Multi-agent system modeling of urban agglomeration's government game and control mechanism: aggregating evolutionary game and small world networks. Chin. J. Syst. Sci. 24(4), 105-110 (2016). in Chinese

24. Luo, H.: Multi-agent simulation experiment of urban agglomeration's government game and control mechanism: investigate the effect of asymmetrical game situation and complementary compensation measures. Chin. J. Syst. Sci. 25(2), 78-83 (2017). in Chinese

25. Luo, H.: How to address multiple sources of influence in group decision-making? In: Morais, D.C., Carreras, A., de Almeida, A.T., Vetschera, R. (eds.) GDN 2019. LNBIP, vol. 351, pp. 17-32. Springer, Cham (2019). https://doi.org/10.1007/9783-030-21711-2_2

26. Luo, H.: Individual, coalitional and structural influence in group decision-making. In: Torra, V., Narukawa, Y., Pasi, G., Viviani, M. (eds.) MDAI 2019. LNCS (LNAI), vol. 11676, pp. 77-91. Springer, Cham (2019). https://doi.org/10.1007/ 978-3-030-26773-5_7

27. Luo, H., Guo, Z., Zhang, Y.: Value analysis of mobile government. Inf. Doc. Serv. 4, 36-40 (2010). in Chinese

28. Luo, H., Meng, Q.: Multi-agent simulation of SC reform and national game. World Econ. Polit. 6, 136-155 (2013). in Chinese 
29. Luo, H., Yang, L.: Measuring power in international organizations: from voting weight to voting power: an analysis of AIIB as an example. World Econ. Polit. 2, 127-154+159-160 (2018). in Chinese

30. Luo, H., Yang, L.: Balance of power and decision-making efficiency in international organizations: an analysis of BRICS new development bank and contingency reserve arrangement as an example. World Econ. Polit. 2, 123-154+159-160 (2019). in Chinese

31. Luo, H., Zhang, Y., Meng, Q.: Modeling and simulation of multi-cities' policy coordination based on MAS. Chin. J. Manag. Sci. 23(1), 89-98 (2015). in Chinese

32. Maran, A., Maudet, N., Pini, M.S., Rossi, F., Venable, K.B.: A framework for aggregating influenced CP-nets and its resistance to bribery. In: Proceedings of the Twenty-Seventh AAAI Conference on Artificial Intelligence, pp. 668-674 (2013)

33. Maudet, N., Pini, M.S., Venable, K.B., Rossi, F.: Influence and aggregation of preferences over combinatorial domains. In: Proceedings of the 11th International Conference on Autonomous Agents and Multiagent Systems, pp. 1313-1314 (2012)

34. Meng, Q., Luo, H.: Modeling and simulation of multi-cities' government collaboration based on MAS: embedded in and feedbacking on heterogeneous social networks. J. Manag. Sci. China 20(3), 183-207 (2017). (in Chinese)

35. Pérez, L.G., Mata, F., Chiclana, F., Gang, K., Herrera-Viedma, E.: Modelling influence in group decision making. Soft Comput. 20(4), 1653-1665 (2016)

36. Purrington, K., Durfee, E.H.: Making social choices from individuals' CP-nets. In: Proceedings of the 6th International Conference on Autonomous Agent and Multiagent System, p. 179 (2007)

37. Salehi-Abari, A., Boutilier, C.: Empathetic social choice on social networks. In: Proceedings of the 13th International Conference on Autonomous Agents and MultiAgent Systems, pp. 693-700 (2014)

38. Wicker, A.W., Doyle, J.: Interest-matching comparisons using CP-nets. In: Proceedings of the Twenty-Second AAAI Conference on Artificial Intelligence, pp. 1914-1915 (2007)

39. Wicker, A.W., Doyle, J.: Comparing preferences expressed by CP-networks. In: Proceedings of the AAAI Workshop on Advances in Preference Handling, pp. 128$133(2008)$ 